
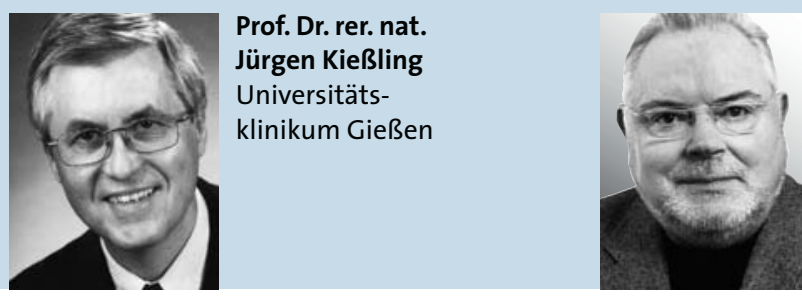

Dr. med.

Dieter Leithäuser

HNO-Arzt, Warburg

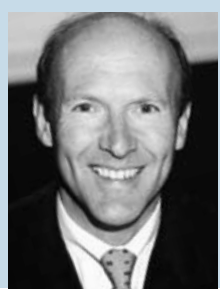

Prof. Dr. med.

Ralph Mösges

Universität Köln

\title{
Cochlea-Implantationen bei Kindern weiterhin sicher
}

\author{
In den USA erhalten aufgrund des dortigen Screenings 85-99\% der Neugeborenen in \\ den ersten Lebenstagen eine Untersuchung auf Schwerhörigkeit. Daher werden immer \\ mehr betroffene Kinder früh erkannt und mit einem Cochlear-Implantat versorgt. \\ Aus diesem Grund überprüften US-Ärzte die Sicherheit des Eingriffs in der eigenen Klinik.
}

$D_{p}^{i}$ Versorgung mit Cochlear-Implantaten bereitet bei Kindern mehrere zusätzliche Probleme, die bei Erwachsenen nicht bestehen. Zu ihnen gehören ein erhöhtes Risiko durch die Anästhesie vor allem im Alter unter sechs Monaten bzw. bei Komorbidität im Alter bis zu einem Jahr, die präoperative Messung des Ausmaßes der Schwerhörigkeit sowie die postoperative Programmierung des Gerätes. Da die Eingriffe immer häufiger erfolgen, haben HNOSpezialisten der Saint Louis University, Missouri, USA, retrospektiv die Daten von 136 Patienten im Alter unter 18 Jahren ausgewertet, die in ihrem Zentrum zur Tertiärversorgung zwischen Januar 2001 und Dezember 2005 ein Cochlear-Implantat erhalten hatten und mindestens drei Jahre nachverfolgt worden waren.

Die meisten Patienten $(60,6 \%)$ hatten einen nicht syndromischen, nicht genetischen, kongenitalen, sensorineuralen Hörverlust. Weitere, seltene Ursachen waren TORCH-Infektionen (toxoplasmosis, other, rubella, cytomegalovirus, herpes: 8,3\%), erweiterter vestibulärer Aquädukt $(6,5 \%)$ und Connexin-Mutationen $(5,8 \%)$. Es gab keine intraoperativen Komplikationen und nur ein Versagen des Gerätes (0,7\%). Die häufigsten Komplikationen waren Flap-Infektionen $(2,6 \%)$ und rasch auftretende postoperative Hämatome (1,9\%). Die Flap-Komplikationen entwickelten sich meist innerhalb von zwei Wochen nach Implantation und konnten alle durch lokale Wundpflege behoben werden.
Fazit: Nach Ansicht der Autoren sind Cochlear-Implantationen bei Kindern demnach weiterhin zuverlässig und sicher, wenn sie von erfahrenen Spezialisten durchgeführt werden. Die Rate schwerer Komplikationen sei gering, solange die Patienten engmaschig kontrolliert würden und die Familien eine angemessene Erwartung hätten. Im Vergleich zu älteren Daten aus anderen Studien scheine die Rate üblicher Komplikationen wie Geräte- oder Flap-Probleme womöglich aufgrund von Verbesserungen in der Technologie und im Follow-up zurückzugehen, doch um dies abzusichern seien weitere Daten notwendig, so die Arbeitsgruppe.

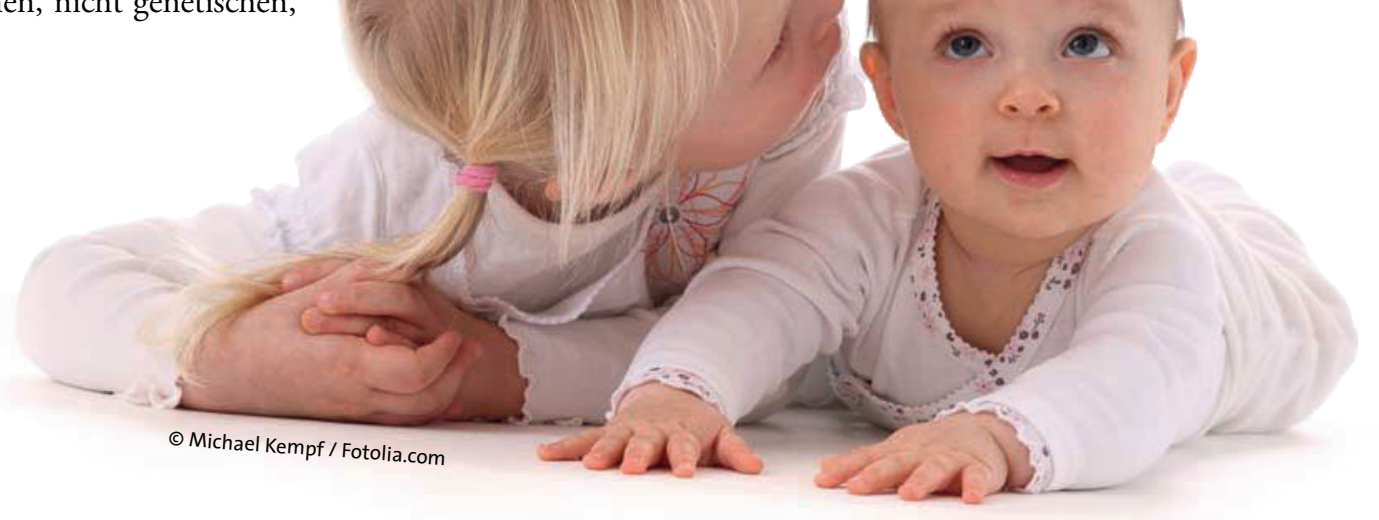

McJunkin J, et al. Complications in pediatric cochlear implants. Am J Otolaryngol. 2010;31(2):110-3 